\title{
Theory of digital magnetoresistance in ferromagnetic resonant-tunneling diodes
}

\author{
Christian Ertler* and Jaroslav Fabian ${ }^{\dagger}$ \\ Institute for Theoretical Physics, University of Regensburg, Universitätsstrasse 31, D-93040 Regensburg, Germany
}

(Received 30 November 2006; published 18 May 2007)

\begin{abstract}
We propose a ferromagnetic spintronic system, which consists of two serial connected resonant-tunneling diodes. One diode is nonmagnetic whereas the other comprises a ferromagnetic emitter and quantum well. Using a self-consistent coherent transport model we show that the current-voltage characteristic of the ferromagnetic diode can be strongly modulated by changing the relative orientation of the magnetizations in the emitter and quantum well, respectively. By a continuous change of the relative magnetization angle the total resistance exhibits a discrete jump realizing digital magnetoresistance. The interplay between the emitter's Fermi energy level and the relative magnetization orientations allows to tailor the current voltage characteristics of the ferromagnetic diode from ohmic to negative differential resistance regime at low voltages.
\end{abstract}

DOI: 10.1103/PhysRevB.75.195323

PACS number(s): 75.50.Pp, 73.40.Gk, 73.21.Fg, 72.25.Dc

\section{INTRODUCTION}

The development of ferromagnetic dilute magnetic semiconductors $(\mathrm{DMSs})^{1-6}$ has opened the possibility of realizing all semiconductor spintronic device concepts, in which the charge current can be modulated by the carriers spin. ${ }^{7}$ For example, spin dependent resonant-tunneling have been investigated both experimentally and theoretically in magnetic double barrier heterostructures with either a ferromagnetic quantum well (QW), e.g., GaMnAs,${ }^{8-15}$ or a paramagnetic QW, which exhibits a giant $g$ factor, e.g., ZnMnSe. ${ }^{16-18}$ By employing resonant interband tunneling an effective injection of spin-polarized electrons into nonmagnetic semiconductors has been demonstrated. ${ }^{19,20}$ Moreover, enhanced tunneling magnetoresistance (TMR) has been predicted and found in double barrier magnetic tunneling junctions, ${ }^{9,14,15,21}$ in which a nonmagnetic QW is sandwiched between two magnetic electrodes. Recently, high magnetocurrents [relative current magnitudes for parallel (P) and antiparallel (AP) orientations of the magnetizations] have been predicted in two coupled magnetic QWs. ${ }^{22}$

Conventional nonmagnetic resonant-tunneling diodes (RTDs) are technologically interesting due to their extreme high speed and low power performance. They allow for simplified circuit concepts based on their specific negative differential resistance (NDR) behavior. ${ }^{23}$ A logic gate named MOBILE (monostable-bistable transition logic element), which consists of two serial connected RTDs, a load and driver, has been proposed and realized by Maezawa and Mizutani. ${ }^{24,25}$ The device is driven by an oscillating input voltage, which performs the transition between the monostable and bistable working point regimes. At low input voltages Kirchoff's laws allow for only one stable dc working point. However, for high input voltages two stable working points become possible due to the $N$-shaped current voltage (IV) characteristic of both the load and the driver RTD. Which of the two working points is actually realized depends on the difference of the load and driver peak currents. When the load peak current is higher than the driver one the working point voltage is high and vice versa. The whole device works actually as a comparator of the load and driver's peak current. For a detailed discussion of the operation principle see Refs. 23-25.
We have recently proposed ${ }^{26}$ that by replacing the driver by a magnetic RTD with a paramagnetic QW the circuit exhibits what we call digital magneto resistance (DMR): the output voltage jumps from low to high after the monostableto-bistable transition if the external magnetic field, which controls the Zeeman splitting in the QW, is higher than some threshold value. The threshold value of the magnetic field can be controlled by a gate voltage, which influences the peak current of the load. The proposed device performs a direct digital conversion of an analog magnetic signal and might be used as a fast magnetic read head. DMR has actually been experimentally demonstrated in an earlier setup by shunting a metallic giant magnetoresistance (GMR) element to a nonmagnetic driver RTD,${ }^{27}$ having the advantage of being nonvolatile upon the loss of power: the state of the device is stored in the magnetization direction of a particular ferromagnetic layer of the GMR element. Such nonvolatile devices are attractive for fast and reliable data storage, e.g, in random access memory applications ${ }^{28}$ or for reprogrammable logics, ${ }^{29}$ in which the logical function of the circuit can be tuned by changing the magnetic state of the device.

In this paper we lay down the physical principles of a nonvolatile ferromagnetic MOBILE. We propose to use a driver RTD, which comprises a ferromagnetic emitter and QW. By performing realistic self-consistent calculations of the $I V$ characteristics DMR is observed when the QW magnetization is tilted. For a proper choice of the emitter's Fermi energy the driver $I V$ can be changed from ohmic to negative differential resistance behavior in the low voltage regime depending on the relative magnetization orientation.

The paper is organized as follows. A discussion of the system and the self-consistent transport model is presented in Sec. II. The simulation results are shown and discussed in detail in Sec. III, and, finally, conclusions are given in Sec. IV.

\section{MODEL}

The operation of the magnetic MOBILE is based on the change of the driver's peak current by applying an external magnetic field or by changing some magnetization direction in the device. In order to realize a nonvolatile ferromagnetic 


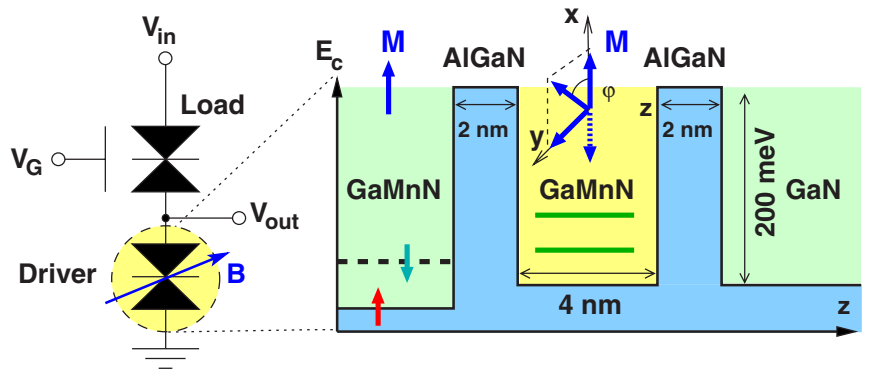

FIG. 1. (Color online) Left: The circuit configuration of the proposed ferromagnetic MOBILE. The load is a conventional RTD, whose peak current can be modified by an external gate voltage $V_{G}$. The driver device consists of a ferromagnetic RTD. The peak current of the driver is controlled by twisting the QW magnetization. Right: The schematic conduction band profile of the ferromagnetic RTD [here made of a $\mathrm{Ga}(\mathrm{Al}, \mathrm{Mn}) \mathrm{N}$ material system] used in the numerical simulations discussed in the text.

MOBILE one can use the double barrier TMR structure, ${ }^{21}$ in which a nonmagnetic QW is sandwiched between two ferromagnetic leads. For low voltages the structure yields large magnetocurrents, but for higher voltages, when the exchange splitting of the collector lead is shifted far below the band edges of the emitter lead by the applied voltage, the magnetocurrent becomes small. This means that for an exchange splitting of the order of a few tens of meV the peak current is hardly influenced by the relative orientation of collector's magnetization, since the peak voltage is already too high. Hence, hardly any DMR would be observed in such a TMR device.

Another setup to realize nonvolatility would be to use a ferromagnetic QW instead of a paramagnetic one. In the proposed paramagnetic MOBILE, ${ }^{26}$ the driver peak current decreases with increasing Zeeman splitting. In ferromagnetic materials it is easier to change the orientation than the magnitude of the magnetization. If the exchange splitting is strongly anisotropic a nonvolatile MOBILE could indeed be realized by a ferromagnetic QW and by rotating its magnetization direction. However, in an isotropic case as considered here an additional ferromagnetic lead is necessary to observe the DMR effect. We consider a ferromagnetic emitter, since a ferromagnetic collector lead would again have little influence on the peak current.

In order to investigate the characteristic physical effects of such a structure as shown in Fig. 1, we consider the emitter and QW to be made of a generic, moderately doped $n$-type ferromagnetic semiconductor. We also expect to observe similar effects in a $p$-type ferromagnetic semiconductor, since in the simplest approach the heavy and light holes can be treated by an effective mass model, ${ }^{30}$ analogous to our description of the conduction electrons here. In todays $p$-type ferromagnetic DMSs, e.g., GaMnAs the Fermi energy cannot be chosen freely, since the ferromagnetic order appears only at high hole densities. ${ }^{31}$ However, for device applications a decoupling of the ferromagnetic order and the doping would be advantageous. There have been several experimental reports on moderately doped $n$-type ferromagnetic semiconductors, e.g., $\mathrm{HgCr}_{2} \mathrm{Se}_{4},{ }^{32} \mathrm{CdCr}_{2} \mathrm{Se}_{4}{ }^{33} \mathrm{CdMnGeP}_{2},{ }^{34}$ and most promising $\mathrm{ZnO}$ and $\mathrm{GaMnN}$.,5,35,36 Many experimental results suggest room temperature (RT) ferromagnetism in transition metal doped $\mathrm{GaN}$ and $\mathrm{ZnO}$. However, it is still controversial if the observed RT ferromagnetism is intrinsic or due to some nonresolved precipitates. Several different mechanisms ${ }^{5,6}$ have been proposed theoretically to be responsible for the observed magnetic hysteresis. A thorough discussion of these issues is given in Refs. 4-6, and references therein.

For the functioning of our proposed ferromagnetic MOBILE we need a conduction band spin splitting of the order of tens of $\mathrm{meV}$, regardless by which mechanism this exchange splitting is induced. In GaMnN it is generally believed that the exchange splitting of the conduction band is about $30-50 \mathrm{meV} .{ }^{37,38}$ In experiments the ferromagnetic order sustains for dopings up to a few $10^{18} \mathrm{~cm}^{-3}$, in thin layers of a few nm width. ${ }^{36}$ It has also been shown theoretically that a small amount of anisotropic coupling in the 2D-Heisenberg model is sufficient to stabilize long range order at finite temperatures. ${ }^{39}$ Two-barrier RTDs based on $n$-GaMnN have already been investigated theoretically. ${ }^{38}$

To be specific, we perform all numerical simulations for a GaMnN quantum well. The circuit diagram of the ferromagnetic MOBILE, the material composition, and the conduction band of the driver RTD is schematically shown in Fig. 1. We consider a two-barrier semiconductor heterostructure, $\mathrm{GaMnN} / \mathrm{Al}_{1-x} \mathrm{Ga}_{x} \mathrm{~N} / \mathrm{GaMnN} / \mathrm{Al}_{1-x} \mathrm{Ga}_{x} \mathrm{~N} / \mathrm{GaN}$, where a $\mathrm{Ga}$ concentration of about $x=17 \%$ is assumed in the barriers yielding a barrier height of about $200 \mathrm{meV} .{ }^{40}$ The QW is undoped, whereas the leads consist of $15 \mathrm{~nm}$ long $n$-doped layers, with $n=2.78 \times 10^{18} \mathrm{~cm}^{-3}$ in the magnetic emitter and $n=2.26 \times 10^{18} \mathrm{~cm}^{-3}$ in the collector lead corresponding to a Fermi energy of $E_{f}=25 \mathrm{meV}$ at the lattice temperature of $T$ $=100 \mathrm{~K}$. The magnetization $\mathbf{M}$ of the lead is considered to be fixed, whereas the ferromagnetic QW is "soft," which means that its magnetization direction can be altered by an external magnetic field.

In order to investigate the IV-characteristics of such a structure we follow the classic treatments of transport in nonmagnetic RTD, ${ }^{41-45}$ where coherent transport in the whole active device region (here the barriers and the $\mathrm{QW}$ ) is assumed. Exploiting the symmetry of the Hamiltonian due to translations in the plane perpendicular to the growth direction $z$ of the heterostructure, the Schrödinger equation can be reduced to a one-dimensional problem. By assigning the spin quantization axis to the fixed magnetization axis of the emitter lead the spinor scattering states $\psi_{\sigma, E}^{j \sigma^{\prime}}(z)$ with the spin quantum number $\sigma= \pm 1 / 2,(\uparrow, \downarrow)$ regarding to the boundary condition of an incident electron with spin $\sigma^{\prime}$ of plane wave form stemming from lead $i[=\operatorname{left}(L)$, right $(R)]$ are then determined in the effective mass envelope function approach by

$$
\left[-\frac{\hbar^{2}}{2} \frac{d}{d z} \frac{1}{m(z)} \frac{d}{d z}+U_{\sigma}(z)\right] \psi_{\sigma}^{i \sigma^{\prime}}(z)=E \psi_{\sigma}^{i \sigma^{\prime}}(z),
$$

with

$$
U_{\sigma}(z)=E_{c}(z)-e \phi(z)+\frac{\Delta_{\mathrm{ex}}(z)}{2} \boldsymbol{\sigma} \cdot \mathbf{e}_{M}(z)
$$

Here, $m$ denotes the effective electron mass, and $E$ is the total longitudinal energy of the electron (the sum of the po- 
tential and longitudinal kinetic energy), $E_{c}(z)$ denotes the intrinsic conduction band profile of the heterostructure, $e$ is the elementary charge, $\phi$ the electrostatic potential, $\Delta_{\text {ex }}$ denotes the exchange splitting of the conduction band, $\boldsymbol{\sigma}$ is the Pauli matrices vector, and $\mathbf{e}_{M}$ is the unit vector of the magnetization. For a realistic simulations space charge effects have to be taken into account. The electrostatic potential $\phi$ is obtained from the Poisson equation

$$
\frac{d}{d z} \epsilon(z) \frac{d}{d z} \phi(z)=\frac{e}{\epsilon_{0}}\left[n(z)-N_{d}(z)\right],
$$

where $\epsilon$ denotes the static dielectric constant, $\epsilon_{0}$ is the permeability of the vacuum, $N_{d}(z)$ is the fixed donor density profile of the device, and $n(z)$ is the electron density. The Poisson equation (2) has to be solved together with the Schrödinger equation (1) in a self-consistent way, since the quantum electron density is given by

$$
n(z)=\frac{1}{4 \pi} \sum_{i, \sigma, \sigma^{\prime}} \int_{U_{i, \sigma^{\prime}}}^{\infty} \mathrm{d} \varepsilon f_{i}(\varepsilon)\left|\psi_{\sigma, \varepsilon}^{j, \sigma^{\prime}}(z)\right|^{2} \frac{1}{\hbar v_{i, \sigma^{\prime}}},
$$

where

$$
f_{i}(\varepsilon)=\frac{m}{\pi \hbar^{2}} k_{B} T \ln \left[1+\exp \left(\mu_{i}-\varepsilon\right)\right] .
$$

Here, $U_{i, \sigma^{\prime}}$ and $v_{i, \sigma^{\prime}}$ denote the spin-dependent potential energy and longitudinal group velocity of the electron in the left and right lead, respectively, $\mu_{i}$ with $\mu_{R}=\mu_{L}-e V_{a}$ is the chemical potential, where we assume that a voltage $V_{a}$ is applied to the right lead, and finally $k_{B}$ labels the Boltzmann constant and $T$ the lattice temperature of the leads. Since we are interested in the generic properties of the proposed structure we neglect the effects of polarization charges at the interfaces, which appear in $\mathrm{GaN}$ due to spontaneous and piezoelectric polarization. ${ }^{40}$

After obtaining the self-consistent potential profile the current density is calculated in the framework of the Landauer-Büttiker formalism. By assuming parabolic bands and using the same effective mass for all layers of the heterostructure, the transmission matrix $T_{\sigma^{\prime} \sigma}$ does not dependent on the transversal kinetic energy of the electrons. Hence, the current density $j_{\sigma^{\prime} \sigma}^{i \rightarrow j}$ regarding to electrons which are incident with spin $\sigma$ from lead $i$ and end up in lead $j$ with spin $\sigma^{\prime}$ can be obtained by generalizing the Tsu-Esaki formula ${ }^{46}$

$$
\underset{j_{\sigma^{\prime} \sigma}^{i \rightarrow j}}{i \rightarrow m}=\frac{e m k_{B} T}{(2 \pi)^{2} \hbar^{3}} \int_{\max \left(U_{i, \sigma}, U_{j, \sigma^{\prime}}\right)}^{\infty} d \varepsilon f_{i}(\varepsilon) T_{\sigma^{\prime} \sigma^{\prime}}^{i \rightarrow j}
$$

According to time-reversal symmetry $T_{\sigma^{\prime} \sigma}^{i \rightarrow j}=T_{\sigma \sigma^{\prime}}^{j \rightarrow i}$ and the total current density is given by

$$
j=\sum_{\sigma \sigma^{\prime}}\left(j_{\sigma^{\prime} \sigma}^{L \rightarrow R}-j_{\sigma \sigma^{\prime}}^{R \rightarrow L}\right),
$$

the difference of left and right flowing currents.

\section{NUMERICS AND SIMULATION RESULTS}

Following Ref. 41, the transmission matrix $T_{\sigma, \sigma^{\prime}}$ is obtained by using the transfer materix approach. The spinor scattering states in Eq. (1) are numerically calculated by applying the fourth-order Runge-Kutta (RK) scheme to the Schrödinger equation. This approach works well for the assumed effective mass model but for more advanced multiband models the transfer matrices can become numerically unstable due to an exponential blowup, requiring improved numerical methods to examine the transport properties of structures with large active regions. ${ }^{47,48}$ In order to investigate the salient features of the proposed structure we furthermore make the widely invoked simplifying assumption that the transmission is independent of the in-plane wave vector of the incident electrons. However, detailed analyses have shown that the in-plane wave vector dependence can significantly affect the $I V$ characteristics leading to a spreading of the transmission maxima. ${ }^{49-51}$ For more quantitative simulations the different in-plane dispersions of all conduction band edges in the structure and, even more relevant, of the subbands in the QW should be taken into account. ${ }^{51}$

Due to the forming of quasibound states in the QW the local density of states (LDOS) is strongly energy dependent. Therefore, we use an adaptive energy mesh for the numerical calculation of the electron density by rewriting the quadrature, Eq. (3), into an initial value problem of an ordinary differential equation and solve it again by applying a fourthorder RK scheme. In this way the computational costs of the numerical integration are strongly reduced (usually 400 energy points are necessary for a relative accuracy of $10^{-4}$, compared to 1200 grid points for an uniform energy mesh). To achieve fast convergence (usually 5-10 iterations steps) between the Schrödinger equations (1) and the Poisson equation (2), we apply a predictor-corrector method. ${ }^{52}$ The guess for the uniformly discretized $(\Delta z=0.1 \mathrm{~nm})$ electrostatic potential is obtained by a Newton-Raphson method, where the necessary Jacobian is estimated by using the semiclassical Thomas-Fermi approximation for the electron density. ${ }^{45}$ To save computational costs the particle density in the leads $n_{i}(z)$ is calculated semiclassically ${ }^{44}$ by

$$
n_{i}(z)=\frac{N_{c}}{2} \sum_{\sigma} \mathcal{F}_{1 / 2}\left[\frac{\mu_{i}-U_{\sigma}(z)}{k_{B} T}\right],
$$

where $N_{c}$ is the effective conduction band density of states and $\mathcal{F}_{1 / 2}$ is the Fermi-Dirac integral of order 1/2. Since the transmission functions are usually "spiky" we use an adaptive Gauss-Kronrod scheme for an efficient numerical calculation of the current density in Eq. (5).

For all simulations we set the Fermi energy to $E_{f}$ $=25 \mathrm{meV}$ (all energies are measured from the unsplit emitter conduction band edge), the lattice temperature to $T=100 \mathrm{~K}$, and we use the same exchange splitting in the QW and the emitter lead, $\Delta_{\mathrm{ex}}=40 \mathrm{meV}$. We assume the spin up conduction band edge to lie energetically higher than the spin down one, which leads to a particle spin polarization of about $-77.6 \%$ in the emitter lead. An effective electron mass of $m / m_{0}=0.228$ (Ref. 40) ( $m_{0}$ denotes the free electron mass) is used and the static dielectric constant is set to $\epsilon=9.5 .^{53}$ 

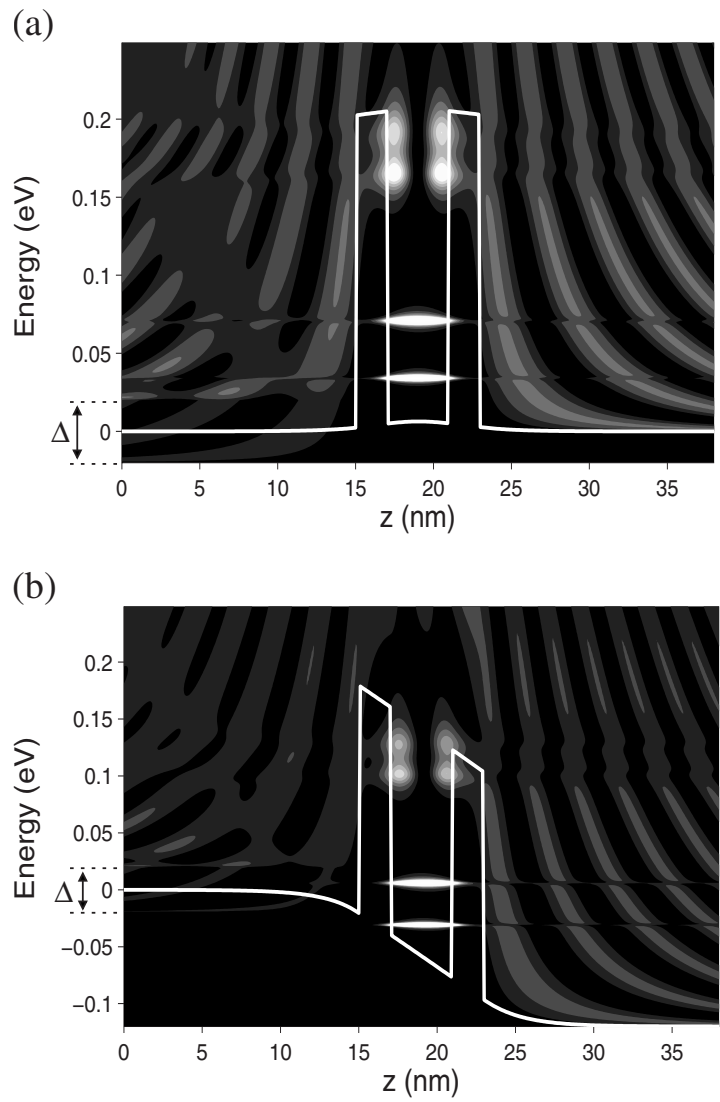

FIG. 2. Contour plots of the local density of states versus energy and growth direction $z$ for parallel magnetization alignment in the case of (a) equilibrium $V_{a}=0$ and (b) at the peak voltage of $V_{a}$ $=0.12 \mathrm{~V}$. The exchange splitting of spin up and down level is clearly visible for the quasibound ground state in the well. The solid lines indicate the self-consistent conduction band profile.

The contour plots of the local density of states (LDOS) of the conduction electrons for $\mathrm{P}$ magnetizations alignment in the considered heterostructure (see Fig. 1) are shown in Fig. 2(a) in the equilibrium case $\left(V_{a}=0\right)$ and (b) at the peak voltage of $V_{a}=0.12 \mathrm{~V}$. The forming of the exchange split spin up and down quasibound states is apparent. In the shown case of $\mathrm{P}$ alignment the spin up level lies energetically higher than the spin down one. The broadening of the first spin resolved quasibound states [at about 34 and $71 \mathrm{meV}$ in Fig. 2(a)] in energy is much smaller than the exchange splitting, whereas for the next higher quasibound states (at about 164 and $190 \mathrm{meV}$ ), which have a node in the middle, both spin levels already overlap. The solid lines indicate the self-consistent conduction band profile. In the self-consistent case parts of the applied voltage already drop before the first barrier but also beyond the second one as can be seen in Fig. 2(b). This is in contrast to the non-self-consistent case, where the applied voltage is assumed to drop linearly only in the active device region, i.e., in the QW and two barriers.

The self-consistent $I V$ characteristics for the structure with the lateral dimensions indicated in Fig. 1 in the case of different relative orientations of the QW magnetization (characterized by the angle $\varphi$ ) is shown in Fig. 3. For comparison, Fig. 4 displays the $I V$ curves for the non-self-

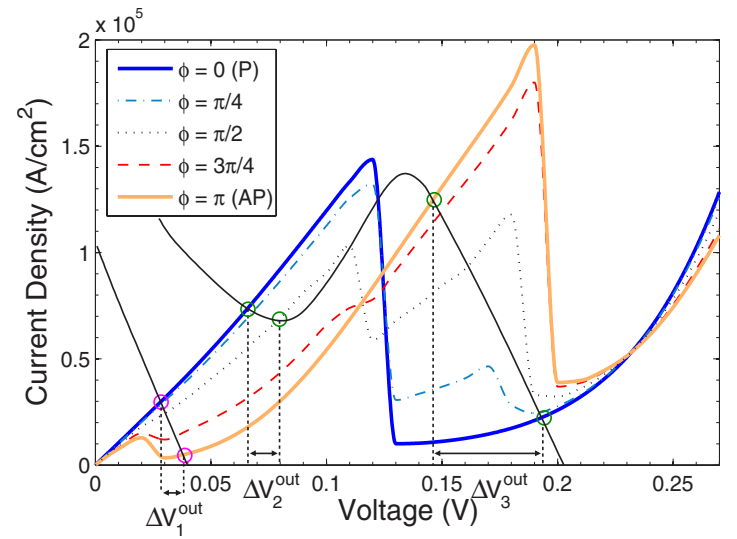

FIG. 3. (Color online) Self-consistent current-voltage characteristics of the magnetic driver-RTD for several relative orientations of the quantum well magnetization (indicated by the angle $\varphi$ ) at the temperature of $T=100 \mathrm{~K}$. The solid black lines show the mirrored $I V$ curve of the load-RTD for low and high input voltages, respectively; working points are indicated by circles. For these fixed low and high input voltages the output voltages are restricted to the intervals $\Delta V_{i}^{\text {out }}, i=1,2,3$.

consistent case. Interestingly, in the latter case there exists a single crossing point for all $I V$ 's with the same current $I_{*}$ at some voltage $V_{*}$. This fact suggests that the $I V$ characteristic for a specific angle $\varphi$ might be written as a simple linear combination of the $\mathrm{P}$ and AP IV's: $I_{\varphi}(V)=a(\varphi) I_{0}(V)$ $+b(\varphi) I_{\pi}(V)$ with $a+b=1$.

The crossing in the non-self-consistent case follows from the linearity of the Schrödinger equation. For the purpose of the proof let us introduce a coordinate system, which we call the " 0 system," where the $z$ axis coincides with the magnetization direction of the QW magnetization and the $y$ axis is given by the growth direction of the heterostructure. It is evident from the symmetry of the structure that instead of twisting the QW magnetization by some angle $-\varphi$ it is equivalent to assume a fixed magnetization direction in the QW and to rotate the emitter's magnetization by the angle $\varphi$.

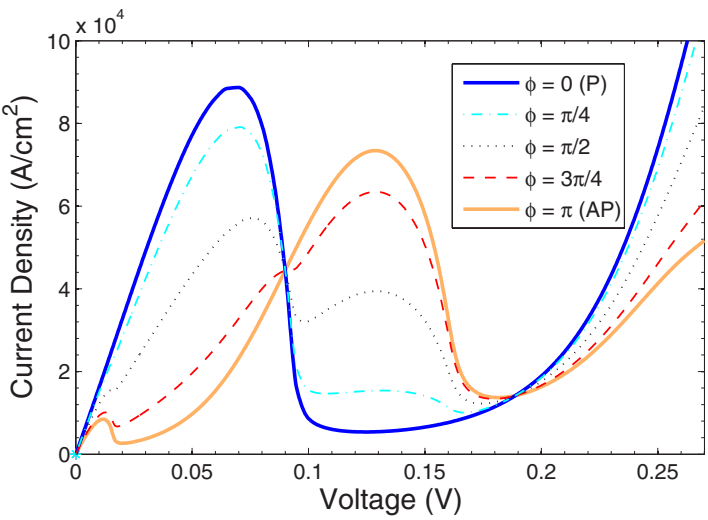

FIG. 4. (Color online) Current-voltage characteristics of the magnetic driver-RTD in the non-self-consistent case for different relative orientations $\varphi$ of the quantum well magnetization at the lattice temperature $T=100 \mathrm{~K}$. The peak voltage and currents are smaller when compared to the self-consistent case of Fig. 3 and there exists a common crossing points for all $I V$ curves. 
In the latter case only the boundary conditions for the Schrödinger equation (1) are changed when compared to the $\mathrm{P}$ alignment. The spinor wave functions in the emitter lead are of the plane-wave form, when they are represented in a coordinate system (the " $\varphi$ system"), where the $z$ axis is given by the emitter's magnetization direction. The " $\varphi$ system" results from the 0 system by rotating the latter around the $y$ axis by the angle $\varphi$. Hence, the spinor representations in the 0 and $\varphi$ systems are connected by $\{\psi\}_{0}=D(-\varphi)\{\psi\}_{\varphi}$, where

$$
D(\varphi)=\left(\begin{array}{cc}
\cos (\varphi / 2) & \sin (\varphi / 2) \\
-\sin (\varphi / 2) & \cos (\varphi / 2)
\end{array}\right) .
$$

Due to the linearity of the Schrödinger equation (1) and since $D(-\varphi)=\cos (\varphi / 2) D(0)-\sin (\varphi / 2) D(\pi)$ we can "divide" the problem into finding the solution for the two boundary conditions $\{\psi\}_{0}=\cos (\varphi / 2)\{\psi\}_{\varphi}$ and $\{\psi\}_{0}=-\sin (\varphi / 2) D(\pi)\{\psi\}_{\varphi}$, respectively. Representing the latter in the $\pi$-coordinate system with its $+z$ axis along the $-z$ axis of the 0 system results in $\{\psi\}_{\pi}=\sin (\varphi / 2)\{\psi\}_{\varphi}$. Thus, we can express the amplitude for the transmission of an incident electron with its spin aligned along the $z$ axis of the $\varphi$ system denoted by $\left|z_{\varphi}, \sigma\right\rangle$ to a right moving plane wave state on the collector side with a spin eigenstate of the $z$ axis of the 0 system $\left|z_{0}, \sigma^{\prime}\right\rangle$ as $\left\langle z_{0}, \sigma^{\prime} \mid z_{\varphi}, \sigma\right\rangle=\cos (\varphi / 2)\left\langle z_{0}, \sigma^{\prime} \mid z_{0}, \sigma\right\rangle+\sin (\varphi / 2)\left\langle z_{0}, \sigma^{\prime} \mid z_{\pi}, \sigma\right\rangle$. Since there is no spin precession for the $\mathrm{P}$ and AP case when the electron transmits the device region and since we assume that spin flipping scattering processes do not occur, the offdiagonal matrix elements of the amplitude matrix $\left\langle z_{0}, \sigma^{\prime} \mid z_{0}, \sigma\right\rangle$ vanish, whereas $\left\langle z_{0}, \sigma^{\prime} \mid z_{\pi}, \sigma\right\rangle=0$ for the diagonal elements. With this the spin-dependent transmission function, which is proportional to the squared transmission amplitude, can be written as

$$
\begin{aligned}
T_{\sigma^{\prime}, \sigma}^{\varphi} \propto\left|\left\langle z_{0}, \sigma^{\prime} \mid z_{\varphi}, \sigma\right\rangle\right|^{2} & \left(\begin{array}{ll}
\cos ^{2}\left(\frac{\varphi}{2}\right)\left|\left\langle z_{0}, \uparrow \mid z_{0}, \uparrow\right\rangle\right|^{2} & \sin ^{2}\left(\frac{\varphi}{2}\right)\left|\left\langle z_{0}, \uparrow \mid z_{\pi}, \downarrow\right\rangle\right|^{2} \\
\sin ^{2}\left(\frac{\varphi}{2}\right)\left|\left\langle z_{0}, \downarrow \mid z_{\pi}, \uparrow\right\rangle\right|^{2} & \cos ^{2}\left(\frac{\varphi}{2}\right)\left|\left\langle z_{0}, \downarrow \mid z_{0}, \downarrow\right\rangle\right|^{2}
\end{array}\right) .
\end{aligned}
$$

Using Eqs. (5) and (6) finally yields the desired result

$$
j_{\varphi}(V)=\cos ^{2}(\varphi / 2) j_{0}(V)+\sin ^{2}(\varphi / 2) j_{\pi}(V) .
$$

This relation is only valid in the non-self-consistent case, since by including space charge effects, the self-consistent electrostatic potential will become, in general, $\varphi$ dependent and the above given considerations break down. We propose that the deviation from a common crossing point $\left(I_{*}, V_{*}\right)$ for all $I V$ 's might be used as a criterion of the relative importance of space charge effects, modulated by $\varphi$, in the device. As can be seen in Fig. 3 no common crossing point appears in the self-consistent case. Since the applied voltage drops over a longer spatial region than in the non-self-consistent case, as can be seen in Fig. 2(b), the self-consistent peak voltages and currents are considerably higher. Hence, for our specific device setup space charge effects strongly influence the obtained $I V$ characteristics.

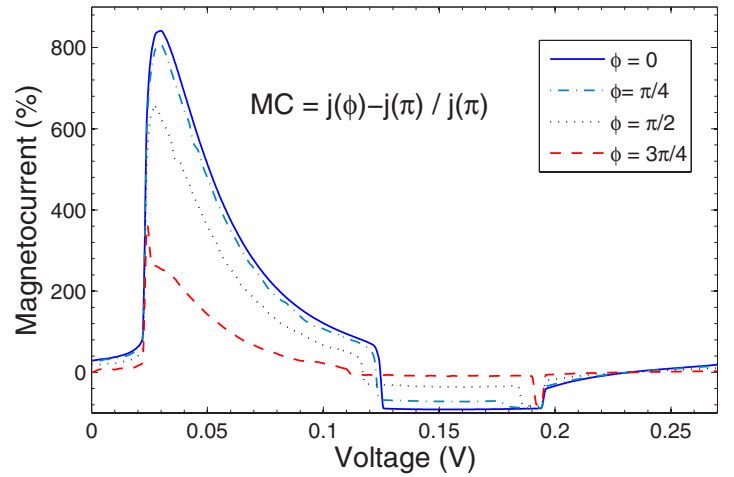

FIG. 5. (Color online) Voltage-dependent magnetocurrent (MC) in the case of different relative orientations $\varphi$ of the magnetization in the quantum well at the temperature of $T=100 \mathrm{~K}$.

The magnetoresistive response is usually expressed in terms of the tunneling magnetoresistance $\mathrm{TMR}=\left(G_{\mathrm{P}}\right.$ $\left.-G_{\mathrm{AP}}\right) / G_{\mathrm{AP}}$, i.e., the relative change of the conductances $G_{\mathrm{P}}$ and $G_{\mathrm{AP}}$ for parallel and antiparallel alignments of the magnetizations. Analogously, one can define a magnetocurrent $M C=\left(I_{\mathrm{P}}-I_{\mathrm{AP}}\right) / I_{\mathrm{AP}}{ }^{7}$ which in our case generalizes to

$$
\operatorname{MC}(\varphi)=\frac{j(\varphi)-j(\pi)}{j(\pi)},
$$

comparing the current densities of a particular magnetization orientation $\varphi$ with the AP alignment. Our self-consistent simulations reveal that the MC increases up to high values of about $800 \%$ (in the case of $\varphi=0$ ) for voltages right after the first NDR region of the AP IV curve ( $V>25 \mathrm{mV}$, see Fig. 5). According to the definition of the MC also negative values can be obtained for high voltages $(V>0.12 \mathrm{~V})$ at which the current is largest for the AP alignment.

Moreover, the current spin polarization with respect to the emitter spin quantization axis is given by

$$
P_{j}=\frac{j_{\uparrow \uparrow}+j_{\uparrow \downarrow}-j_{\downarrow \uparrow}-j_{\downarrow \downarrow}}{j_{\uparrow \uparrow}+j_{\uparrow \downarrow}+j_{\downarrow \uparrow}+j_{\downarrow \downarrow}} .
$$

As shown in Fig. 6, $P_{j}$ is strongly modulated when the QW magnetization is flipped from $\mathrm{P}$ to AP. At low voltages the polarization can be continuously changed from $-93 \%$ for the $\mathrm{P}$ alignment up to $+90 \%$ for the AP orientation. Hence, in the low voltage regime the ferromagnetic $\mathrm{QW}$ acts as a spin aligner. Interestingly, in the AP case the current polarization sharply slopes from +90 to $-98 \%$ over the small first NDR voltage interval, which allows us to use the device also as a voltage controlled spin switcher.

To illustrate our results we introduce a model, which allows to give a qualitative estimate of the $I V$ curves for the extreme cases of parallel (P) $\varphi=0$ and antiparallel (AP) $\varphi$ $=\pi$ magnetization orientation. In particular, the model allows for a better understanding for the influence of the following three simulation parameters: (i) the energy difference for the lowest spin up and down quasi bound states $\delta=E_{\uparrow}-E_{\downarrow}$ [it should be noted that $\delta=37 \mathrm{meV}$ is a bit smaller than the exchange splitting of the conduction band in the QW $\left(\Delta_{\mathrm{ex}}\right.$ $=40 \mathrm{meV}$ ) due to the finite barrier height], (ii) the exchange 


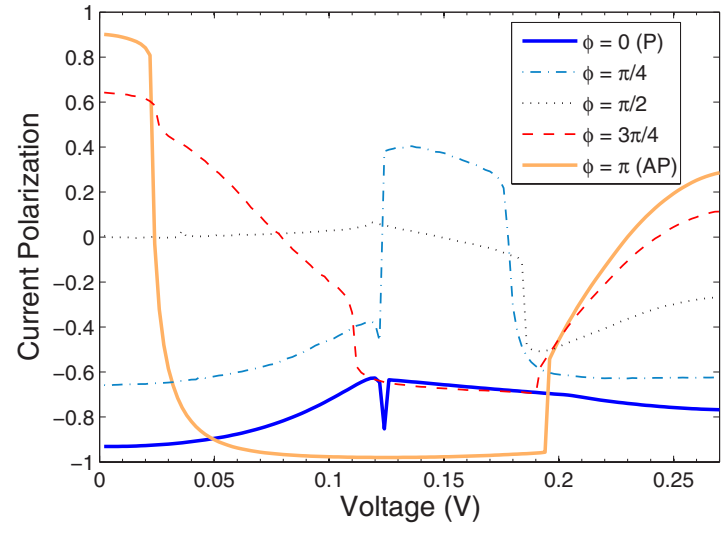

FIG. 6. (Color online) Current spin polarization as a function of the applied voltage for different orientations of the quantum well magnetization (indicated by angle $\varphi$ ) at the lattice temperature of $T=100 \mathrm{~K}$.

splitting $\Delta$ in the emitter lead, and (iii) the relative position of the Fermi energy level $E_{f}$ in the emitter conduction band. By applying a voltage to the collector lead the quasibound states $E_{\sigma}$ are shifted to lower energies. At first glance the voltage dependence of quasibound energy levels can be described by

$$
E_{\sigma}(V)=E_{\sigma}(0)-\alpha V
$$

where the parameter $\alpha$ is assumed to be voltage-independent $(\alpha=1 / 2$ for the linear voltage drop and usually $\alpha<1 / 2$ for the self-consistent case). For the following discussion it is more convenient to consider that the magnetization of the QW is fixed and, hence, in the AP case the magnetization of the emitter is flipped. This leads to completely identical $I V$ characteristics since in the AP case only the sign of the spin polarization of the current is changed but not its magnitude. The model is schematically illustrated in Fig. 7.

At low temperatures current can flow only if the quasibound states are dropped below the emitter Fermi energy level, hence, the spin up and down currents are switched on at the voltages $V_{\sigma}^{\text {on }}=\frac{1}{\alpha}\left(E_{\sigma}-E_{f}\right)$. These spin-polarized currents are switched off, if the corresponding quasibound states are shifted below the emitter spin up and down conduction band edges, which leads to $V_{\sigma, \text { off }}^{\mathrm{P}}=\frac{1}{\alpha}\left(E_{\sigma}-\sigma \Delta / 2\right)$ for the $\mathrm{P}$ alignment and $V_{\sigma, \text { off }}^{\mathrm{AP}}=\frac{1}{\alpha}\left(E_{\sigma}+\sigma \Delta / 2\right)$ for the $\mathrm{AP}$ case, respectively. For higher temperatures the "switching on" relations will be thermally smeared on the order of a few $k_{B} T$ around the Fermi energy but the "switching off" relations are still valid, as long as inelastic scattering can be neglected as assumed in our coherent transport model. From these relations it immediately follows that $V_{\downarrow, \text { off }}^{\mathrm{AP}}<V_{\uparrow, \text { off }}^{\mathrm{P}} \leq V_{\downarrow, \text { off }}^{\mathrm{P}}<V_{\uparrow, \text { off }}^{\mathrm{AP}}$ for $\delta \leq \Delta$ and $V_{\downarrow \text {,off }}^{\mathrm{AP}}<V_{\downarrow, \text { off }}^{\mathrm{P}}<V_{\uparrow, \text { off }}^{\mathrm{P}}<V_{\uparrow, \text { off }}^{\mathrm{AP}}$ for $\delta>\Delta$, respectively. In the case of $\delta \leq \Delta$ the distances between these "off-switching" voltages are illustrated in Fig. 7(b); for $\delta>\Delta$ they are just given by interchanging $(\delta \leftrightarrow \Delta)$.

First of all this model reveals that the peak voltage for the $\mathrm{AP}$ alignment is always higher than in the $\mathrm{P}$ case, since $V_{\uparrow, \text { off }}^{\mathrm{AP}}>V_{\sigma, \text { off }}^{\mathrm{P}}$, as obtained in our simulated $I V$ curves, Fig. 3 . For the special case of $\delta=\Delta$, which is approximately fulfilled

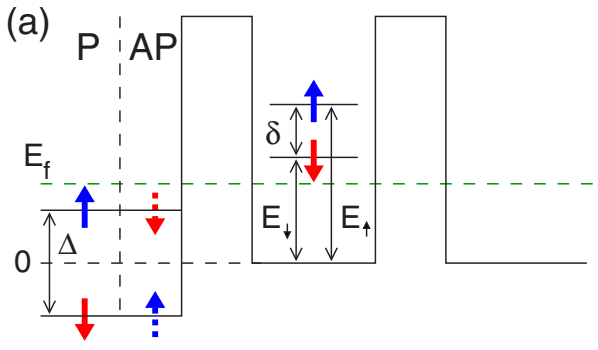

(b)

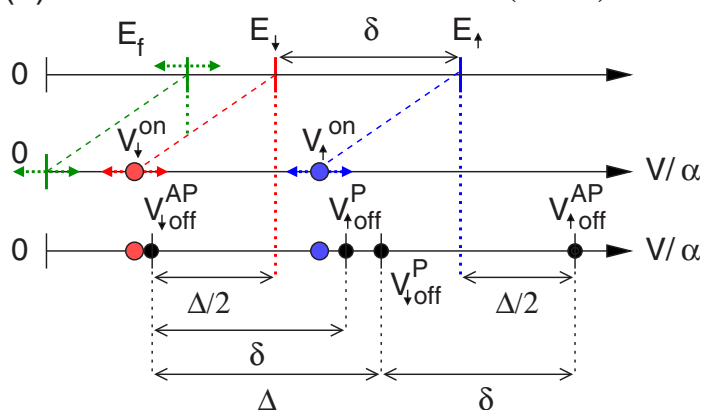

FIG. 7. (Color online) Illustration of the analytic model discussed in the text for the case of parallel (P) and antiparallel (AP) magnetization alignment. In the model the magnetization of the emitter is "soft," whereas the QW magnetization is assumed to be fixed. Part (a) shows the spin resolved conduction band profile in the emitter with the spin splitting $\Delta$, the first quasibound states $E_{\uparrow}$ and $E_{\downarrow}$ in the quantum well energetically separated by $\delta$, and the emitter Fermi energy level $E_{f}$. Part (b) illustrates graphically the relative positions of the emitter Fermi and well energies (top axis) and of the so-called "on" and "off" switching voltages introduced in the model (middle and bottom axis) on a $1 / \alpha$ voltage scale with the parameter $\alpha$ defined in Eq. (12). The on-switching voltages (lightcolored circles) $V_{\uparrow, \downarrow}^{\text {on }}=\left(E_{\uparrow, \downarrow}-E_{f}\right) / \alpha$ are defined as voltages at which the well states start to become resonant and the off-switching voltages (blacked filled circles) denote the voltages at which the well states are pushed below the emitters spin up and down conduction band, respectively, for both the $\mathrm{P}$ and AP cases. The relative position between the on- and off-switching voltages can be changed by moving the Fermi energy level.

in our simulations, the spin up and down current in the P case can be switched off at the same voltage. The relative position of the "on-switching" voltages on the voltage scale [indicated by the colored circles in Fig. 7(b)] can be changed by the position of the Fermi energy level. By an appropriate choice of $E_{f}$, as indicated in Fig. 7(b), in the AP case the spin down current can be switched off before the spin up current is switched on. This leads to the NDR behavior in the low voltage regime, and the sharp current polarization drop. For the $\mathrm{P}$ alignment the total current is in the whole voltage range dominated by the spin down component. At low voltages up to $40 \mathrm{mV}$ almost all current "flows" through the spin down channel but then also the spin up components starts to contribute to the total current, diminishing the spin current polarization. Since both spin currents are "switched off" at almost the same voltage in the $\mathrm{P}$ case the $I V$ curve is nearly ohmic in the low voltage regime. This simple discussion shows that rotating the QW magnetization can drastically change the $I V$ curve. 
For the "intermediate" case of perpendicular orientation $(\varphi=\pi / 2)$ (see Fig. 3) two peak voltages appear with nearly the same values as those obtained for the P and AP alignment, respectively. However, most important for observing DMR is that the peak current is already remarkably reduced when the relative magnetization orientation is tilted, say by an angle of a few tens of degrees, out of the P alignment. If we assume the load peak current is smaller than the driver peak current in the $\mathrm{P}$ case, the monostable-to-bistable transition results in a low output voltage. However, if we tilt the magnetization orientation, the load and driver peak current become equal at some angle $\varphi_{\text {th }}$, which can be called the "threshold angle." For $\varphi>\varphi_{\text {th }}$ a high output voltage is obtained in the bistable regime. Hence, the output voltage suddenly jumps from low to high after performing the monostable-to-bistable transition, effectively realizing DMR. The threshold angle can be controlled indirectly by properly tuning the load peak current, which is altered by an external gate voltage applied to the load device. Assuming a fixed low and high input voltage the output voltage is restricted to three different voltage intervals $\Delta V_{i}^{\text {out }}, i=1,2,3$ as illustrated in Fig. 3. The high voltage interval $\Delta V_{3}^{\text {out }}$ is considerably separated from the low voltages intervals $\Delta V_{1}^{\text {out }}$ and $\Delta V_{2}^{\text {out }}$. This allows for a direct digital detection of the tilted $\mathrm{QW}$ magnetization.

The question of how fast the monostable-to-bistable transition can be performed is closely connected to the subtle and still not clarified problem of determining the error rates in MOBILEs. The tilting of the QW magnetization leads to a redistribution of the quasibound states, which usually takes place on the time scale of the order of a hundred of femtoseconds. The switching time of RTDs, however, is limited by the "classical" RC time constant, which is typically of the order of a few picoseconds. ${ }^{54}$ In experiments the conventional MOBILEs randomly jump between high and low output voltage in the transition region and an erroneous transition can occur due to parasitic capacitances or external electrical noise. ${ }^{55}$ Transient studies of conventional MOBILEs based on an equivalent circuit model have shown that an error-free transitions with clock rise times on the order of the RC time of the RTD are possible if the output capacitance $C_{\text {out }}<(k-1) C_{\mathrm{RTD}},{ }^{56}$ where $C_{\mathrm{RTD}}$ is an average capacitance of the RTD and $k$ is the ratio of load to driver peak current. Recently, conventional MOBILEs have been demonstrated to work up to frequencies as high as $100 \mathrm{GHz}$ by employing a symmetric clock configuration for the input voltage. ${ }^{57}$ This gives reason for a possible application of the proposed ferromagnetic MOBILE as a very fast "readout" of magnetically stored information.

Figure 8 shows a blow up of the self-consistent $I V$ characteristics of Fig. 3 for low voltages. By rotating the QW magnetization the $I V$ curves change from ohmic to NDR behavior. As illustrated in the inset of Fig. 8 the spin down current becomes off resonance already before the current can flow "through" the spin up quasibound state leading effectively to NDR. This interesting behavior can be used to perform the monostable-to-bistable transition by twisting the QW magnetization instead of changing the input voltage from low to high as discussed above. For this, we assume to use a linear load resistance instead of the load-RTD in the

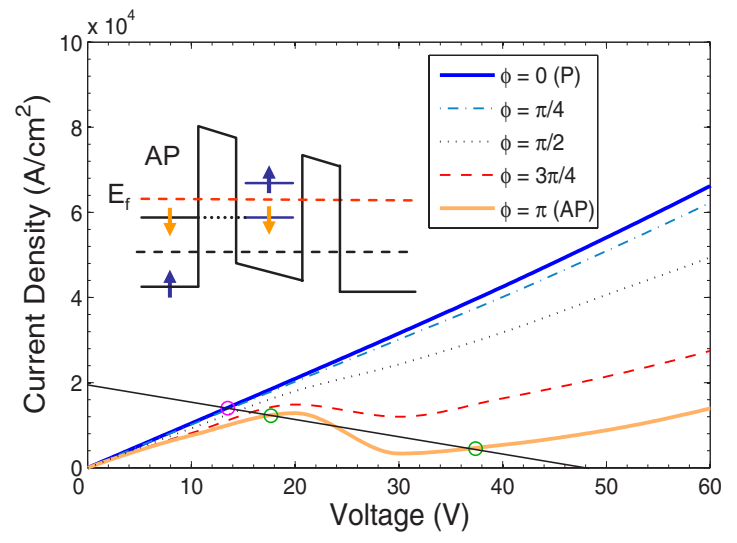

FIG. 8. (Color online) Blow up of the self-consistent currentvoltage characteristics displayed in Fig. 3 for low voltages. By replacing the load-RTD by a linear load resistance (indicated by the black solid line) allows us to perform the monostable-to-bistable transition by flipping the QW magnetization from $\mathrm{P}$ to AP. The working points are indicated by circles. The inset schematically shows the arrangement of the quasibound levels at the peak voltage in the AP case.

circuit setup of Fig. 1. For an appropriately high input voltage two stable working points are obtained in the AP case, whereas only one crossing point appear in the load line diagram for the P alignment (see Fig. 8). With this again DMR can be realized as follows. Let us assume that at the beginning the circuit operates at the high voltage working point of the AP orientation. If we tilt the QW magnetization, suddenly at some threshold angle, the circuit is switched from the bistable to the monostable regime, leading to a discrete jump from high to low output voltage. This allows us to "digitally" detect a disturbance of the AP magnetization alignment. After this detection and after the recovering of the AP orientation the circuit will end up in the low voltage state of the bistable regime. By applying a small current pulse to the circuit it can again be reset to the initial high voltage state. Another application could be a memory cell, in which the binary information is stored in the $\mathrm{P}$ and AP configuration and is read out by a small current pulse, which leads in the $\mathrm{AP}$ case to a voltage swing to the high voltage state.

\section{CONCLUSIONS}

We have proposed a ferromagnetic MOBILE, where the driver-RTD comprises a ferromagnetic emitter and QW. By using a self-consistent coherent transport model we have shown that by changing the relative orientation of the two magnetizations the $I V$ characteristics are strongly modulated and that nonvolatile DMR can be realized with this circuit. In particular, this allows for an electrical and direct digital detection of a small distortion out of the $\mathrm{P}$ alignment. The comparison of the self-consistent with the non-selfconsistent model, where a linear voltage drop is assumed in the device, reveals that space charge effects have to be included to get more realistic $I V$ characteristics. High MCs up to $800 \%$ are obtained in the AP case, at $100 \mathrm{~K}$, and the current spin polarization can be continuously changed from 
+90 to $-93 \%$ by either flipping the QW magnetization or by altering the applied voltage for the AP alignment. By a proper choice of the Fermi energy level and the magnitudes of the exchange splitting in both the emitter and QW, respectively, the $I V$ curves can be changed in the low voltage regime from ohmic to NDR behavior by twisting the QW magnetization from $\mathrm{P}$ to AP alignment. When serially connected to a load resistance this allows us to accomplish a monostable-to-bistable working point transition just by flipping the QW magnetization, in contrast to the usual way of performing the transition where the input voltage is in- creased from low to high level. Since conventional MOBILEs have been demonstrated to work up to $100 \mathrm{GHz}$ the proposed device might be useful for performing very fast detections of magnetic signals and for realizing fast magnetic random access memories.

\section{ACKNOWLEDGMENTS}

This work has been supported by the Deutsche Forschungsgemeinschaft SFB 689. The authors thank S. J. Pearton and K. Maezawa for valuable discussions.
*Email address: christian.ertler@physik.uni-regensburg.de

†Email address: jaroslav.fabian@ physik.uni-regensburg.de

${ }^{1}$ H. Ohno, Science 281, 951 (1998).

${ }^{2}$ T. Dietl, Semicond. Sci. Technol. 17, 377 (2002).

${ }^{3}$ T. Dietl, Modern Aspects of Spin Physics (Springer, Berlin, 2006), Chap. Semiconductor Spintronics, pp. 1-46.

${ }^{4}$ S. J. Pearton, C. R. Abernathy, D. P. Norton, A. F. Hebard, Y. D. Park, L. A. Boatner, and J. D. Budai, Mater. Sci. Eng., R. 40, 137 (2003).

${ }^{5}$ C. Liu, F. Yun, and H. Morkoç, J. Mater. Sci.: Mater. Electron. 16, 555 (2005).

${ }^{6}$ T. Jungwirth, J. Sinova, J. Mašek, J. Kučera, and A. H. MacDonald, Rev. Mod. Phys. 78, 809 (2006).

${ }^{7}$ I. Žutić, J. Fabian, and S. Das Sarma, Rev. Mod. Phys. 76, 323 (2004).

${ }^{8}$ A. G. Petukhov, D. O. Demchenko, and A. N. Chantis, J. Vac. Sci. Technol. B 18, 2109 (2000).

${ }^{9}$ T. Hayashi, M. Tanaka, and A. Asamitsu, J. Appl. Phys. 87, 4673 (2000).

${ }^{10}$ A. Oiwa, R. Moriya, Y. Kashimura, and H. Munekata, J. Magn. Magn. Mater. 276, 2016 (2004).

${ }^{11}$ J. Furdyna, T. Wojtowicz, X. Liu, K. M. Yu, W. Walukiewicz, I. Vurgaftman, and J. R. Meyer, J. Phys.: Condens. Matter 16, S5499 (2004)

${ }^{12}$ N. Lebedeva and P. Kuivalainen, Phys. Status Solidi B 242, 1660 (2005).

${ }^{13}$ S. Ganguly, L. F. Register, S. Banerjee, and A. H. MacDonald, Phys. Rev. B 71, 245306 (2005).

${ }^{14}$ S. Ohya, P. N. Hai, and M. Tanaka, Appl. Phys. Lett. 87, 012105 (2005).

${ }^{15}$ R. Mattana, M. Elsen, J.-M. George, H. Jaffrès, F. Nguyen van Dau, A. Fert, M. F. Wyczisk, J. Olivier, P. Galtier, B. Lépine, A. Guivarc'h, and G. Jézéquel, Phys. Rev. B 71, 075206 (2005).

${ }^{16}$ T. Gruber, M. Keim, R. Fiederling, G. Reuscher, W. Ossau, G. Schmidt, L. W. Molenkamp, and A. Waag, Appl. Phys. Lett. 78, 1101 (2001).

${ }^{17}$ A. Slobodskyy, C. Gould, T. Slobodskyy, C. R. Becker, G. Schmidt, and L. W. Molenkamp, Phys. Rev. Lett. 90, 246601 (2003).

${ }^{18}$ N. N. Beletskii, G. P. Berman, and S. A. Borysenko, Phys. Rev. B 71, 125325 (2005).

${ }^{19}$ A. G. Petukhov, D. O. Demchenko, and A. N. Chantis, Phys. Rev. B 68, 125332 (2003).

${ }^{20}$ I. Vurgaftman and J. R. Meyer, Phys. Rev. B 67, 125209 (2003).
${ }^{21}$ A. G. Petukhov, A. N. Chantis, and D. O. Demchenko, Phys. Rev. Lett. 89, 107205 (2002).

${ }^{22}$ C. Ertler and J. Fabian, Appl. Phys. Lett. 89, 242101 (2006).

${ }^{23} \mathrm{~K}$. Maezawa and A. Förster, Nanoelectronics and Information Technology (Wiley-VCH, New York, 2003), Chap. Quantum Transport Devices Based on Resonant Tunneling, pp. 407-424.

${ }^{24}$ K. Maezawa and T. Mizutani, Jpn. J. Appl. Phys., Part 2 32, L42 (1993).

${ }^{25}$ K. Maezawa, T. Akeyoshi, and T. Mizutani, IEEE Trans. Electron Devices 41, 148 (1994).

${ }^{26}$ C. Ertler and J. Fabian, Appl. Phys. Lett. 89, 193507 (2006).

${ }^{27}$ A. Hanbicki, R. Magno, S. F. Cheng, Y. D. Park, A. S. Bracker, and B. T. Jonker, Appl. Phys. Lett. 79, 1190 (2001).

${ }^{28}$ J. M. Daughton, J. Magn. Magn. Mater. 192, 334 (1999).

${ }^{29}$ T. Whitaker, Compound Semicond. 1, 36 (1998).

${ }^{30}$ E. E. Mendez, W. I. Wang, B. Ricco, and L. Esaki, Appl. Phys. Lett. 47, 415 (1985).

${ }^{31}$ T. Dietl, H. Ohno, and F. Matsukura, Phys. Rev. B 63, 195205 (2001).

${ }^{32}$ V. V. Osipov, N. A. Viglin, and A. A. Samokhvalov, Phys. Lett. A 247, 353 (1998).

${ }^{33}$ Y. D. Park, A. T. Hanbicki, J. E. Mattson, and B. T. Jonker, Appl. Phys. Lett. 81, 1471 (2002).

${ }^{34}$ G. A. Medvedkin, T. Ishibashi, T. Nishi, K. Hayata, Y. Hasegawa, and K. Sato, Jpn. J. Appl. Phys., Part 2 39, L949 (2000).

${ }^{35}$ G. T. Thaler, M. E. Overberg, B. Gila, R. Frazier, C. R. Abernathy, S. J. Pearton, J. S. Lee, S. Y. Lee, Y. D. Park, Z. G. Khim, J. Kim, and F. Ren, Appl. Phys. Lett. 80, 3964 (2002).

${ }^{36}$ S. J. Pearton, C. R. Abernathy, G. T. Thaler, R. Frazier, F. Ren, A. F. Hebard, Y. D. Park, D. P. Norton, W. Tang, M. Stavola, J. M. Zavada, and R. G. Wilson, Physica B 34039 (2003).

${ }^{37}$ S. J. Pearton (private communication).

${ }^{38}$ M. K. Li, N. M. Kim, S. J. Lee, H. C. Jeon, and T. W. Kang, Appl. Phys. Lett. 88, 162102 (2006).

${ }^{39}$ D. J. Priour, E. H. Hwang Jr., and S. Das Sarma, Phys. Rev. Lett. 95, 037201 (2005).

${ }^{40}$ O. Ambacher, J. Majewski, C. Miskys, A. Link, M. Hermann, M. Eickhoff, M. Stutzmann, F. Bernardini, V. Fiorentini, V. Tilak, B. Schaff, and L. F. Eastman, J. Phys.: Condens. Matter 14, 3399 (2002).

${ }^{41}$ M. O. Vassell, J. Lee, and H. F. Lockwood, J. Appl. Phys. 54, 5206 (1983).

${ }^{42}$ M. Cahay, M. McLennan, S. Datta, and M. S. Lundstrom, Appl. Phys. Lett. 50, 612 (1987). 
${ }^{43}$ W. Pötz, J. Appl. Phys. 66, 2458 (1989).

${ }^{44}$ H. Ohnishi, T. Inata, S. Muto, N. Yokoyama, and A. Shibatomi, Appl. Phys. Lett. 49, 1248 (1986).

${ }^{45}$ R. Lake, G. Klimeck, R. C. Bowen, and D. Jovanovic, J. Appl. Phys. 81, 7845 (1997).

${ }^{46}$ R. Tsu and L. Esaki, Appl. Phys. Lett. 22, 562 (1973).

${ }^{47}$ T. B. Boykin, J. P. A. van der Wagt, and J. S. Harris, Phys. Rev. B 43, 4777 (1991).

${ }^{48}$ D. Z.-Y. Ting, E. T. Yu, and T. C. McGill, Phys. Rev. B 45, 3583 (1992).

${ }^{49}$ M. S. Kiledjian, J. N. Schulman, K. L. Wang, and K. V. Rousseau, Phys. Rev. B 46, 16012 (1992).

${ }^{50}$ T. B. Boykin, Phys. Rev. B 51, 4289 (1995).

${ }^{51}$ R. C. Bowen, G. Klimeck, R. K. Lake, W. R. Frensley, and T.
Moise, J. Appl. Phys. 81, 3207 (1997).

${ }^{52}$ A. Trellakis, A. T. Galick, A. Pacelli, and U. Ravaioli, J. Appl. Phys. 81, 7880 (1997).

${ }^{53}$ K. F. Brennan and A. S. Brown, Theory of Modern Electronic Semiconductor Devices (Wiley, New York, 2002).

${ }^{54}$ S. K. Diamond, E. Özbay, M. J. W. Rodwell, D. M. Bloom, Y. C. Pao, and J. Harris, Appl. Phys. Lett. 54, 153 (1989).

${ }^{55} \mathrm{~K}$. Maeazawa (private communication).

${ }^{56}$ K. Maezawa, Jpn. J. Appl. Phys., Part 1 34, 1213 (1995).

${ }^{57}$ K. Maezawa, H. Sugiyama, S. Kishimoto, and T. Mizutani, Proceedings of the International Conference on Indium Phosphide and Related Materials, May 7-11, 2006 (IEEE, New York, 2006), pp. 46-49. 